\title{
Order-Preserving Variants of the Basic Principles of Functional Analysis
}

\author{
A. A. Zaitov \\ Tashkent institute of architecture and civil engineering, Tashkent, Uzbekistan
}

\section{Article Info \\ Keywords: Functional analysis, Order- preserving functional, Vector space with an order unit 2010 AMS: 46B40, 46T99, 47 HO7}

Received: 27 December 2018

Accepted: 20 February 2019

Available online: 17 June 2019

\begin{abstract}
We will establish order-preserving versions of the basic principles of functional analysis such as Hahn-Banach, Banach-Steinhaus, open mapping, and Banach-Alaoglu theorems.
\end{abstract}

\section{Introduction}

Recently researches in the field of idempotent mathematics and also Choquet integrals intensively develop. Since its introduction in 1974 by Sugeno, the concept of fuzzy measure has been often used in multicriteria decision making. Later in [1], the authors explained the methodology of using the Choquet integral in multicriteria decision making. The notion of idempotent measure (Maslov integral) finds important applications in different part of mathematics, fuzzy topology, mathematical physics, and economics (see the article [2] and the bibliography therein). As well known idempotent measures and Choquet integrals are weakly additive, order-preserving functionals. But for this functionals there not establish yet the basic principles (analogous principles of Functional Analysis, see, for example, [3]). In the present paper, we will establish order-preserving versions of the basic principles of Functional Analysis such as the Hahn-Banach, Banach-Steinhaus, open mapping and Banach-Alaoglu theorems. Recently, in [4] the uniform boundedness principle for nonlinear operators on cones of functions was investigate. In works [5]-[7] by the author announced open mapping theorem for order-preserving operators and Banach-Alaoglu theorem for order-preserving functionals, in particular case, on the function spaces.

Remind that partially ordered vector space is a pair $(L, \leqslant)$ where $L$ is a vector space over the field $\mathbb{R}$ of real numbers, $\leqslant$ is an order satisfying the following conditions:

1) if $x \leqslant y$, then $x+u \leqslant y+u$ for all $x, y, u \in L$;

2) if $x \leqslant y$, then $\lambda x \leqslant \lambda y$ for all $x, y \in L$ and $\lambda \in \mathbb{R}_{+}$.

If the conditions 1 ) and 2) hold then they say that $\leqslant$ is a linear order. A formation of a vector space $L$ with a linear order $\leqslant$ over $\mathbb{R}$ is equivalent to indicate a set $L_{+} \subset L$ called a positive cone in $L$ and owning the properties:

$$
L_{+}+L_{+} \subset L_{+} ; \quad \lambda L_{+} \subset L_{+}, \quad \lambda \in \mathbb{R}_{+} ; \quad L_{+} \cap\left(-L_{+}\right)=\{0\} .
$$

In this case, the order $\leqslant$ and the positive cone $L_{+}$are connected by a relation

$$
x \leqslant y \Leftrightarrow y-x \in L_{+}, \quad x, y \in L .
$$

Elements of $L_{+}$is called positive vectors of $L$.

Let $\left(L, L_{+}\right)$be a partially ordered vector space. We say [8] that $L_{+}$is full (or that $L_{+}$is a full cone) if $L=L_{+}-L_{+}$.

Let $L$ be a partially ordered vector space over the field $\mathbb{R}$ of real numbers, and $L_{+}$be a full cone in it. Let $x_{1}, x_{2} \in L$ be arbitrary various points. The set $\left[x_{1}, x_{2}\right]=\left\{\alpha x_{1}+(1-\alpha) x_{2}: \alpha \in[0,1]\right\}$ is called a segment connecting points $x_{1}$ and $x_{2}$. A point $x \in\left[x_{1}, x_{2}\right]$ is an inner point of the segment $\left[x_{1}, x_{2}\right]$ if $x_{1} \neq x \neq x_{2}$. 
Let $x \in L_{+}$. The point $x$ is said to be an inner point of the cone $L_{+}$if for any segment $\left[x_{1}, x_{2}\right]$ containing $x$ as an inner point, the segment $\left[x_{1}, x_{2}\right] \cap L_{+}$also contains it as an inner point. The set of all inner points of the cone $L_{+}$is called an interior of this cone, and it denotes as $\operatorname{Int} L_{+}$.

Fix an inner point $x_{0} \in L_{+}$. For a $\delta>0$ we determine a $\delta$-neighbourhood (with respect to the cone $L_{+}$and the point $x_{0}$ ) of zero $0 \in L$ as following:

$$
\langle 0 ; \delta\rangle=\left\{x \in L:\left(\delta x_{0} \pm x\right) \in \operatorname{Int} L_{+}\right\} .
$$

It is easy to see that a family of the sets of the view (1.1) forms a base of neighbourhoods of zero. A neighbourhood of an arbitrary point $z \in L$ can be defined by the shifts of the neighbourhoods of zero:

$$
\langle z ; \delta\rangle=\langle 0 ; \delta\rangle+z=\{x+z \in L: x \in\langle 0 ; \delta\rangle\}=\left\{x+z \in L:\left(\delta x_{0} \pm x\right) \in \operatorname{Int} L_{+}\right\}=\left\{y \in L:\left(\delta x_{0} \pm(y-z)\right) \in \operatorname{Int} L_{+}\right\} .
$$

Proposition 1.1. A collection

$$
\{\langle z ; \delta\rangle: z \in L, \delta>0\}
$$

forms a base of a Hausdorff topology on L. Further, L equipped with this topology becomes a topological vector space.

Proof. The proof consists of direct checking.

An element $1 \in L$ of a partially ordered vector space $L$ is called (strongly) order unit if $L=\bigcup_{n=1}^{\infty}[-n 1, n 1]$. This is equivalent to what for every $x \in L$ there exists $\lambda>0$ such that $-\lambda 1 \leqslant x \leqslant \lambda 1$. Let $x \in L$. A partially ordered vector space $L$ is called Archimedean if the inequality $n x \leqslant 1$ executed for all $n=1,2, \ldots$, implies $x \leqslant 0$. In this case on $L$ one can define a norm by the equality

$$
\|x\|=\inf \{\lambda>0:-\lambda 1 \leqslant x \leqslant \lambda 1\} .
$$

The obtained norm is said to be an order norm. A partially ordered vector space $L$ is called a vector space with an order unit if $L$ has an order unit and $L$ is an Archimedean space. A topology on $L$ generated by the norm (1.2) is called order (vector) topology. For a subset $X \subset L$ by Int $X$ we denote the interior of $X$ according to the order topology on $L$. We accept the following agreement

$$
x<y \Leftrightarrow y-x \in \operatorname{Int} L_{+} .
$$

A set $U\left(0_{E}, \varepsilon\right)=\left\{x \in E:-\varepsilon 1_{E}<x<\varepsilon 1_{E}\right\}$ is an open neighbourhood of zero $0_{E}$ concerning to the order topology. As vector topology is translation invariance then for every point $x \in E$ a set $U(x, \varepsilon)=\left\{y \in E:-\varepsilon 1_{E}<y-x<\varepsilon 1_{E}\right\}$ is an open neighbourhood of $x$ with respect to the order topology.

Proposition 1.2. The order topology and the topology introduced by Proposition 1.1 on a vector space with an order unit coincide.

Proof. Proof is trivial.

\section{Extensions of order-preserving functionals}

In this section, we will prove the order-preserving functional's variant of the Hahn-Banach theorem, one of the basic principles of functional analysis.

Definition 2.1. A subset $B$ of a partially ordered vector space $L$ is said to be an A-subspace concerning to a fixed inner point $x_{0}$ of a full cone $L_{+} \subset L$ if $0 \in B$, and $x \in B$ implies $\left(x+\lambda x_{0}\right) \in B$ for each $\lambda \in \mathbb{R}$.

The following assertion is evident.

Lemma 2.2. A subspace $B$ of the partially ordered vector space $L$ is an A-subspace according to $x_{0}$ iff it contains $x_{0}$.

Note that the space $L$ and its subspace $\left\{\lambda x_{0}: \lambda \in \mathbb{R}\right\}$ are trivial $A$-subspaces. As distinct from the linear case the set $\{0\}$ is not $A$-subspace. It is easy to see that an intersection of any collection of $A$-subspaces is a $A$-subspace. In particular, an intersection of all $A$-subspaces containing a given set $X$ is the minimal $A$-subspace, containing $X$; this $A$-subspace we call as $a$ weakly additive span of $X$, and designate through $A(X)$. The following statement describes a structure of the weakly additive span of a given set.

Proposition 2.3. A weakly additive span $A(X)$ of a subset $X$ of a partially ordered linear space L consists of a (set-theoretic) union of $\left\{\lambda x_{0}: \lambda \in \mathbb{R}\right\}$ and the collection of all sums of the look $x+\lambda x_{0}, x \in X, \lambda \in \mathbb{R}$, i.e.

$$
A(X)=\left\{\lambda x_{0}: \lambda \in \mathbb{R}\right\} \cup \bigcup_{\substack{x \in X, \lambda \in \mathbb{R}}}\left\{x+\lambda x_{0}\right\}=\bigcup_{\substack{x \in X \cup\{0\}, \lambda \in \mathbb{R}}}\left\{x+\lambda x_{0}\right\},
$$

in particular, if $x_{0} \in X$ then

$$
A(X)=\bigcup_{\substack{x \in X, \lambda \in \mathbb{R}}}\left\{x+\lambda x_{0}\right\}
$$

Proof. The proof is obvious. 
Let's denote

$$
\Lambda=\left\{\lambda x_{0} ; \lambda \in \mathbb{R}\right\}
$$

Then we have

$$
A(X)=\bigcup_{x \in X \cup\{0\}}(x+\Lambda) .
$$

The last equality explains the name ' $A$-subspace'. Every $A$-subspace $A(X)$ consists of the union of one-dimensional subspace $\Lambda \subset L$ and affine subsets $x+\Lambda \subset L, x \in X$.

Definition 2.4. A functional $f: L \rightarrow \mathbb{R}$ is called:

1) weakly additive (according to the point $x_{0}$ ) if

$$
f\left(x+\lambda x_{0}\right)=f(x)+\lambda f\left(x_{0}\right), \quad x \in L, \quad \lambda \in \mathbb{R} ;
$$

2) order-preserving (concerning to the cone $L_{+}$) if for every pair $x, y \in L$ belonging $y-x \in L_{+}$implies the inequality

$$
f(x) \leqslant f(y)
$$

3) normed (with respect to the point $\left.x_{0}\right)$ if $f\left(x_{0}\right)=1$.

From the definition immediately follows that weakly additive functional is linear on the one-dimensional subspace $\left\{\lambda x_{0} ; \lambda \in \mathbb{R}\right\}$ of $L$. From here we have $f(0)=0$.

Let $\left(L, L_{+}\right)$be a partially ordered real vector space. A functional $f: L \rightarrow \mathbb{R}$ is called positive if $f\left(L_{+}\right) \subseteq[0,+\infty)$. Each weakly additive, order-preserving functional is positive. Really, let $x \in L_{+}$. Then $x-0 \in L_{+}$. Since $f$ is order-preserving functional, then $f(x) \geqslant f(0)$. Consequently, $f(x) \geqslant 0$. There exists a function which is weakly additive, positive but does not order-preserving.

Example 2.5. Let $L=\mathbb{R}^{2}=\left\{\left(x_{1}, x_{2}\right): x_{i} \in \mathbb{R}, i=1,2\right\}$ be a partially ordered vector space with respect to the usual linear operations ' ' the multiplication by real numbers, ' + ' - the sum of elements of $L$, and to the pointwise order $\leqslant$ on $L$, which defines as $\left(x_{1}, x_{2}\right) \leqslant\left(y_{1}, y_{2}\right) \Leftrightarrow$ $x_{1} \leqslant y_{1}$ and $x_{2} \leqslant y_{2}$. The set $L_{+}=\left\{\left(x_{1}, x_{2}\right) \in \mathbb{R}^{2}: x_{i} \geqslant 0, i=1,2\right\}$ is a full positive cone in $L$. Fix an inner point $\mathbf{1}=(1,1) \in L_{+}$and define a functional $f: \mathbb{R}^{2} \rightarrow \mathbb{R}$ by the rule

$$
f\left(x_{1}, x_{2}\right)=\frac{1}{2}\left(x_{1}+x_{2}+\sqrt{\left|x_{2}-x_{1}\right|}\right), \quad\left(x_{1}, x_{2}\right) \in \mathbb{R}^{2} .
$$

It is clear that $f$ is a positive functional. Moreover, for every $\left(x_{1}, x_{2}\right) \in \mathbb{R}^{2}$ and $\lambda \in \mathbb{R}$ we have

$$
\begin{aligned}
f\left(x_{1}+\lambda, x_{2}+\lambda\right)=\frac{1}{2}\left(\left(x_{1}+\lambda\right)+\left(x_{2}+\lambda\right)+\sqrt{\left|\left(x_{2}+\lambda\right)-\left(x_{1}+\lambda\right)\right|}\right) & = \\
& =\frac{1}{2}\left(x_{1}+x_{2}+\sqrt{\left|x_{2}-x_{1}\right|}\right)+\lambda=f\left(x_{1}, x_{2}\right)+\lambda f(1,1)
\end{aligned}
$$

i.e. $f$ is a weakly additive (according to the inner point $(1,1)$ ) functional. But we have $f\left(\frac{1}{2}, \frac{1}{2}\right)<f\left(\frac{1}{4}, \frac{1}{2}\right)$ though $\left(\frac{1}{2}, \frac{1}{2}\right)-\left(\frac{1}{4}, \frac{1}{2}\right) \in L_{+}$. Thus, $f$ is weakly additive, positive but does not order-preserving functional.

Proposition 2.6. If an order-preserving, weakly additive functional $f: L \rightarrow \mathbb{R}$ is continuous at zero 0 then it is continuous on the whole $L$.

Proof. Let for every $\varepsilon>0$ there exists $\delta>0$ such that $|f(x)|<\varepsilon$ for all $x \in\langle 0 ; \delta\rangle \subset L$. Let $y \in L$ be an arbitrary nonzero element. Consider a neighbourhood

$$
\left\langle y ; \frac{\delta}{2}\right\rangle=\left\{z \in L:\left(\frac{\delta}{2} x_{0} \pm(z-y)\right) \in \operatorname{Int} L_{+}\right\}
$$

For every $z \in\left\langle y ; \frac{\delta}{2}\right\rangle$ we have:

1) $f\left(\frac{\delta}{2} x_{0}\right)+f(y) \geqslant f(z)$ since $\left(\frac{\delta}{2} x_{0}+y\right)-z \in \operatorname{Int} L_{+}$;

2) $f\left(\frac{\delta}{2} x_{0}\right)+f(z) \geqslant f(y)$ since $\left(\frac{\delta}{2} x_{0}+z\right)-y \in \operatorname{Int} L_{+}$.

From here follows that

$$
|f(z)-f(y)| \leqslant f\left(\frac{\delta}{2} x_{0}\right) .
$$

Now we take $x \in L$ such that $x-\frac{\delta}{2} x_{0} \in L_{+}$and $\frac{3 \delta}{4} x_{0}-x \in L_{+}$. Then $f\left(\frac{\delta}{2} x_{0}\right) \leqslant f(x)$ and $f(x) \leqslant f\left(\frac{3 \delta}{4} x_{0}\right)$. On the other hand $f\left(\frac{3 \delta}{4} x_{0}\right)<\varepsilon$ so far as $\frac{3 \delta}{4} x_{0} \in\langle 0 ; \delta\rangle$. Consequently $|f(z)-f(y)|<\varepsilon$ for each $z \in\left\langle y ; \frac{\delta}{2}\right\rangle$. So $f$ is continuous at $y \in L$. Thus $f$ is continuous on the whole $L$ owing to arbitrariness of $y \in L$.

A weakly additive, order-preserving functional $f: L \rightarrow \mathbb{R}$ is called bounded if $\sup \{|f(x)|: x \in\langle 0 ; 1\rangle\}<\infty$.

Proposition 2.7. A weakly additive, order-preserving functional is bounded if and only if it is continuous. 
Proof. Let $f: L \rightarrow \mathbb{R}$ be weakly additive, order-preserving bounded functional. Let $f\left(x_{0}\right)=a<\infty$ and $\delta x_{0} \pm(z-y) \in \operatorname{Int} L_{+}$. Then similarly to (2.1) one can show that $|f(z)-f(y)|<\delta f\left(x_{0}\right)=\delta a$, and consequently $f$ is continuous.

Conversely, let a weakly additive, order-preserving functional $f: L \rightarrow \mathbb{R}$ be continuous. Then there exists $\delta>0$ such that $|f(x)|<1$ at all $x \in\langle 0 ; \delta\rangle$. In particular, $\left|\frac{\delta}{2} f\left(x_{0}\right)\right|<1$ so far as $\frac{\delta}{2} x_{0} \in\langle 0 ; \delta\rangle$. Hence, $\left|f\left(x_{0}\right)\right|<\frac{2}{\delta}<\infty$, i.e. $\sup \{|f(x)|: x \in\langle 0 ; 1\rangle\}<\frac{2}{\delta}<\infty$.

Corollary 2.8. A weakly additive, order-preserving, normed functional is continuous (or, the same, bounded).

The following statement is an analog of Hahn-Banach theorem for weakly additive, order-preserving functionals.

Theorem 2.9. Let $B$ be an A-subspace of the space $L$. Then for every weakly additive, order-preserving functional $f: B \rightarrow \mathbb{R}$ there exists $a$ weakly additive, order-preserving functional $f_{0}: L \rightarrow \mathbb{R}$ such that $\left.f_{0}\right|_{B}=f$.

Proof. Let $y \in L \backslash B$. Put $B^{\prime}=B \cup\left\{y+\lambda x_{0}: \lambda \in \mathbb{R}\right\}$. Obviously that $B^{\prime}$ is an $A$-subspace of $L$. Put

$$
B^{+}=\{z \in B: z-y \in B\} \quad \text { and } \quad B^{-}=\{z \in B: y-z \in B\} .
$$

The obtained sets $B^{+}$and $B^{-}$are not empty. Indeed, take $\lambda>0$ such that $y \in\langle 0 ; \lambda\rangle$. Then evidently that $2 \lambda x_{0} \in B^{+}$and $-2 \lambda x_{0} \in B^{-}$. Put

$$
p^{+}=\inf \left\{f(z): z \in B^{+}\right\}, \quad p^{-}=\sup \left\{f(z): z \in B^{-}\right\} .
$$

We have $p^{-} \leqslant p^{+}$. Indeed, $y-z_{1} \in L_{+}$if provided $z_{1} \in B^{-}$, and $z_{2}-y \in L_{+}$if provided $z_{2} \in B^{+}$. From here we get $z_{2}-z_{1} \in L_{+}$. Consequently $f\left(z_{1}\right) \leqslant f\left(z_{2}\right)$ for all $z_{1} \in B^{-}$and $z_{2} \in B^{+}$, i.e. $p^{-} \leqslant p^{+}$. Take a number $p$ such that $p^{-} \leqslant p \leqslant p^{+}$and put

$$
f^{\prime}\left(y+\lambda x_{0}\right)=p+\lambda f\left(x_{0}\right) .
$$

In such a way we define an extension $f^{\prime}$ of $f$ from $B$ on $B^{\prime}$. From the definition directly implies that $f^{\prime}$ is a weakly additive functional. We will show that $f^{\prime}$ is order-preserving. It is order-preserving on $B$ owing to $f^{\prime} \mid B=f$. Besides it is evident that $f^{\prime}$ is order-preserving on $\left\{y+\lambda x_{0}: \lambda \in \mathbb{R}\right\}$. Let now $z-\left(y+\lambda x_{0}\right) \in L_{+}$, where $z \in B$. Then $\left(z-\lambda x_{0}\right)-y \in L_{+}$, i.e. $\left(z-\lambda x_{0}\right) \in B^{+}$. That is why

$$
f^{\prime}\left(z-\lambda x_{0}\right)=f\left(z-\lambda x_{0}\right)=f(z)-\lambda f\left(x_{0}\right) \geqslant p^{+} \geqslant p=f^{\prime}(y),
$$

i.e. $f^{\prime}(z) \geqslant f^{\prime}\left(y+\lambda x_{0}\right)$. In the case when $\left(y+\lambda x_{0}\right)-z \in L_{+}$one can similarly show that $f^{\prime}(z) \leqslant f^{\prime}\left(y+\lambda x_{0}\right)$.

Thus, a weakly additive, order-preserving continuous functional $f: B \rightarrow \mathbb{R}$ defining on an $A$-subspace $B$ can be extended to a weakly additive, order-preserving continuous functional $f^{\prime}: B^{\prime} \rightarrow \mathbb{R}$ on a wider $A$-subspace $B^{\prime}$ of $L$. At the same time the equality $f^{\prime}\left(x_{0}\right)=f\left(x_{0}\right)$ holds.

Consider the set of all pairs $\left(B^{\prime}, f^{\prime}\right)$ such that $B \subset B^{\prime} \subset L$ where $B^{\prime}$ is an $A$-subspace, $f^{\prime}: B^{\prime} \rightarrow \mathbb{R}$ is a weakly additive, order-preserving continuous extension of $f$. The relation $\left(B^{\prime}, f^{\prime}\right) \leqslant\left(B^{\prime \prime}, f^{\prime \prime}\right)$ meaning that $f^{\prime \prime}: B^{\prime \prime} \rightarrow \mathbb{R}$ is a weakly additive, order-preserving continuous extension of $f^{\prime}$ on a subspace $B^{\prime \prime}, B^{\prime} \subset B^{\prime \prime} \subset L$, turns this set into a partially ordered set in which all chains are bounded. By Zorn's lemma, there is the maximal element $\left(B_{0}, f_{0}\right)$ of this set. We will show that $B_{0}=L$.

Suppose that $B_{0} \neq L$. Take any point $y \in L \backslash B_{0}$ and put $B_{1}=B_{0} \cup\left\{y+\lambda x_{0}: \lambda \in \mathbb{R}\right\}$. Then $f_{0}$ can be extended to $f_{1}: B_{1} \rightarrow \mathbb{R}$, and consequently, $\left(B_{0}, f_{0}\right) \leqslant\left(B_{1}, f_{1}\right)$. We got a contradiction with maximality of $B_{0}$. So, $B_{0}=L$.

\section{Uniform boundedness principle for order-preserving operators}

Let $(E, \leqslant)$ and $(F, \leqslant)$ be partially ordered vector spaces.

Definition 3.1. A map $T: E \rightarrow F$ is said to be an order-preserving operator if for arbitrary points $x, y \in E$ the inequality $x \leqslant y$ implies $T(x) \leqslant T(y)$.

Let $(E, \leqslant)$ be a partially ordered vector space with an order unit $1_{E}$ and $(F, \leqslant)$ be a partially ordered vector space.

Definition 3.2. A map $T: E \rightarrow F$ is said to be a weakly additive operator if $T\left(x+\lambda 1_{E}\right)=T(x)+\lambda T\left(1_{E}\right)$ takes place for each $x \in E$ and $\lambda \in \mathbb{R}$.

The last definition immediately implies $T\left(0_{E}\right)=T\left(1_{E}-1_{E}\right)=T\left(1_{E}\right)-T\left(1_{E}\right)=0_{F}$, i.e. $T\left(0_{E}\right)=0_{F}$ for a weakly additive operator $T: E \rightarrow F$.

The following statement shows weakly additive, order-preserving operators of vector spaces with an order unit are automatical continuous.

Proposition 3.3. If $E$ and $F$ are vector spaces with an order unit then each weakly additive, order-preserving operator $T: E \rightarrow F$ is continuous.

Proof. We will show the operator $T$ is continuous at zero $0_{E}$. At first we note the following case. If $T\left(1_{E}\right)=0_{F}$ then $T(x)=0_{F}$ for all $x \in E$ since $T$ is a weakly additive and order-preserving operator, and for every $x$ there exists $\lambda>0$ such that $-\lambda 1_{E} \leqslant x \leqslant \lambda 1_{E}$. So $T(E) \subset\left\{0_{F}\right\}$. This case we will not consider, i.e. suppose $T\left(1_{E}\right) \neq 0_{F}$. Then $\left\|T\left(1_{E}\right)\right\| \neq 0$.

Let $V\left(0_{F}, \varepsilon\right)=\left\{y \in F:-\varepsilon 1_{F}<y<\varepsilon 1_{F}\right\}$ be a neighbourhood of zero $0_{F}$ in $F$, where $\varepsilon>0$. Take the neighbourhood $U\left(0_{E}, \frac{\varepsilon}{\left\|T\left(1_{E}\right)\right\|}\right)$ of zero $0_{E}$ in $E$. For each vector $x \in U$ we have $-\frac{\varepsilon}{\left\|T\left(1_{E}\right)\right\|} 1_{E}<x<\frac{\varepsilon}{\left\|T\left(1_{E}\right)\right\|} 1_{E}$. Then $-\frac{\varepsilon}{\left\|T\left(1_{E}\right)\right\|} T\left(1_{E}\right)<T(x)<\frac{\varepsilon}{\left\|T\left(1_{E}\right)\right\|} T\left(1_{E}\right)$ since $T$ is a weakly additive, order-preserving operator. From here we get $\|T(x)\|<\varepsilon$, i.e. $T(U) \subset V$. Thus $T$ is continuous at $0_{E}$.

The remaining part of the proof is similar to the Proof of Proposition 2.6. 
Remark 3.4. It is obvious that each linear non-negative operator on spaces with an order unit is weakly additive and order-preserving. The converse, in general, is not true. But, nevertheless, such operators are linear on a one-dimensional subspace $\left\{\lambda 1_{E}: \lambda \in \mathbb{R}\right\} \subset E$. In this case the image of the subspace $\left\{\lambda 1_{E}: \lambda \in \mathbb{R}\right\}$ under the map $T$ is, as clearly, a one-dimensional subspace $\left\{\lambda T\left(1_{E}\right): \lambda \in \mathbb{R}\right\} \subset F$. We have $T\left(1_{E}\right) \in F_{+}$but it is optional $T\left(1_{E}\right) \in \operatorname{Int} F_{+}$. Therefore $T\left(1_{E}\right)$ is an order unit in $T(E)$ but it is optional to be an order unit in $F$. From here and Proposition 3.3 follows that for every weakly additive, order-preserving operator $T: E \rightarrow F$ on spaces $E, F$ with an order unit the inequality $\left\|T\left(1_{E}\right)\right\|<\infty$ takes place.

Remind the following notions. A set $A$ in a normed space $E$ is called bounded if there exists $R>0$ such that $A$ can be placed into the ball $\{x \in E:\|x\| \leqslant R\}$. A map $T: E \rightarrow F$ of normed spaces is called bounded if it carries over a bounded set in $E$ to a bounded set in $F$. It is obvious that the boundedness of the map $T$ is equivalent to limitation of the set $\{\|T(x)\|: x \in E,\|x\| \leqslant R\}$ for every $R>0$. In other words, $\sup \{\|T(x)\|: x \in E,\|x\| \leqslant R\}<\infty$ for every bounded map $T$ and for each $R>0$.

The following statement shows weakly additive, order-preserving operators of vector spaces with an order unit are automatical bounded.

Proposition 3.5. Each weakly additive, order-preserving operator $T: E \rightarrow F$ of spaces with an order unit is bounded.

Proof. The proof follows from Remark 3.4.

Let $E$ and $F$ be vector spaces with an order unit, $1_{E}$ and $1_{F}$, respectively. A collection $\mathscr{H}$ of weakly additive, order-preserving operators $T: E \rightarrow F$ is said to be equicontinuous if to every neighbourhood $V$ of zero in $F$ there corresponds a neighbourhood $U$ of zero in $E$ such that $T(U) \subset V$ for all $T \in \mathscr{H}$. If the collection $\mathscr{H}$ consists only one weakly additive, order-preserving operator $T$, then $\mathscr{H}$ is equicontinuous as $T$ is continuous, and $\mathscr{H}$ is uniformly bounded owing to boundedness of $T$. The following statement shows that each equicontinuous collection of weakly additive, order-preserving operators on vector spaces with an order unit is uniformly bounded.

Proposition 3.6. Let $E$ and $F$ be vector spaces with an order unit, $\mathscr{H}$ an equicontinuous collection of weakly additive, order-preserving operators from $E$ into $F$, and $A$ a bounded subset of $E$. Then $F$ has a bounded subset $B$ such that $T(A) \subset B$ for every $T \in \mathscr{H}$.

Proof. Put $B=\bigcup_{T \in \mathscr{H}} T(A)$. Since the collection $\mathscr{H}$ is equicontinuous then for every neighbourhood $V=V\left(0_{F}, \varepsilon\right)$ of zero in $F$ there exists a neighbourhood $U=U\left(0_{E}, \delta\right)$ of zero in $E$ that $T(U) \subset V$ for all $T \in \mathscr{H}$. So far as $A$ is bounded for enough big $t \in \mathbb{R}$ we have $A \subset t U$. It is clear, that $T(A) \subset T(t U)$. Assume that $x \in t U$. Then $\|x\|<t \delta$, i.e. $-t \delta 1_{E}<x<t \delta 1_{E}$. As $T$ is weakly additive and order-preserving we have $-t \delta T\left(1_{E}\right)<T(x)<t \delta T\left(1_{E}\right),\|T(x)\|<t \delta\left\|T\left(1_{E}\right)\right\|$, consequently, $\left\|\frac{1}{t} T(x)\right\|<\delta\left\|T\left(1_{E}\right)\right\|=\left\|T\left(\delta 1_{E}\right)\right\| \leqslant \varepsilon$. Hence, $T(t U) \subset t V$. Thus $T(A) \subset t V$ for all $T \in \mathscr{H}$. It means that $B \subset t V$, i.e. the set $B$ is bounded.

The following result is a weakly additive, order-preserving operators' variant of the Banach-Steinhaus theorem.

Theorem 3.7. Let $E$ and $F$ be vector spaces with an order unit, $\mathscr{H}$ be a collection of weakly additive, order-preserving operators $T: E \rightarrow F$, and $A$ be a set consisting of all points $x \in E$ whose orbits $\mathscr{H}(x)=\{T(x): T \in \mathscr{H}\}$ are bounded in $F$. If $A$ is a set of the second category then $A=E$ and the collection $\mathscr{H}$ is equicontinuous.

Proof. Let $V=V\left(0_{F}, \varepsilon\right)$ and $W=W\left(0_{F}, \varepsilon^{\prime}\right)$ be neighbourhoods such that $\bar{V}+\bar{V} \subset W$ where $\bar{V}$ is the closure of $V$ with respect to order topology in $F$. Put $B=\bigcap_{T \in \mathscr{H}} T^{-1}(\bar{V})$. Let $x \in A$. Then for some positive integer $n$ we have $\mathscr{H}(x) \subset n V$ by virtue of boundedness of $\mathscr{H}(x)$. Hence $T(x) \in n V$ or $x \in n T^{-1}(V)$ for all $T \in \mathscr{H}$. It means that $x \in n B$. Thus $A \subset \bigcup_{n=1}^{\infty} n B$. Thence at least one of the sets $n B$ is the second category owing to $A$ is so. A map $x \mapsto n x$ is a homeomorphism $E$ onto itself. Consequently the set $B$ is the second category. Continuity of operators $T \in \mathscr{H}$ implies $B$ is closed in $E$. As $B$ is the second category set, it has an inner point. By the construction of $B$ one can see that $\delta 1_{E}$ lies in $B$ as an inner point for enough small $\delta \in \mathbb{R}$. Let $\delta 1_{E}$ be such an inner point in $B$. Then a set $B-\delta 1_{E}=\left\{x-\delta 1_{E}: x \in B\right\}$ contains some neighborhood $U=U\left(0_{E}, \delta^{\prime}\right)$ of zero and

$$
T(U) \subset T\left(B-\delta 1_{E}\right)=\left\{T\left(x-\delta 1_{E}\right): x \in B\right\}=\left\{T(x)-\delta T\left(1_{E}\right): x \in B\right\}=T(B)-\delta T\left(1_{E}\right) \subset \bar{V}-\bar{V} \subset W
$$

for all $T \in \mathscr{H}$. It means that $\mathscr{H}$ is a equicontinuous collection. Then $\mathscr{H}$ is uniform bounded by Proposition 3.6. That is why an orbit $\mathscr{H}(x)$ is bounded for each $x \in E$. Consequently, since $A$ consists of points of $E$ whose orbits $\mathscr{H}(x)=\{T(x): T \in \mathscr{H}\}$ are bounded in $F$ we have $E \subset A$. Therefore $A=E$.

If a vector space with an order unit is a Banach space with respect to order norm then it said to be a complete space with an order unit. As each Banach space is a set of the second category then Theorem 3.7 directly implies

Corollary 3.8. Let $E$ be a complete space with an order unit and $F$ a vector space with an order unit, $\mathscr{H}$ a collection of weakly additive, order-preserving operators $T: E \rightarrow F$, and a collection $\mathscr{H}(x)=\{T(x): T \in \mathscr{H}\}$ bounded in $F$ for each $x \in E$. Then $\mathscr{H}$ is an equicontinuous collection.

As Proposition 3.6 holds then Corollary 3.8 means that pointwise boundedness of an arbitrary collection weakly additive, order-preserving operators from a complete space with an order unit into a vector space with an order unit implies uniform boundedness of this collection. Let $E$ and $F$ be vector spaces with an order unit, $\left\{T_{n}\right\}$ a sequence of weakly additive, order-preserving operators $T_{n}: E \rightarrow F$. If for every $x \in E$ there exists a limit $\lim _{n \rightarrow \infty} T_{n}(x)$ then putting

$$
T(x)=\lim _{n \rightarrow \infty} T_{n}(x), \quad x \in E,
$$

we have a weakly additive, order-preserving operator. Indeed,

$$
T\left(x+\lambda 1_{E}\right)=\lim _{n \rightarrow \infty} T_{n}\left(x+\lambda 1_{E}\right)=\lim _{n \rightarrow \infty}\left(T_{n}(x)+\lambda T_{n}\left(1_{E}\right)\right)=T(x)+\lambda T\left(1_{E}\right),
$$


and if $x \leqslant y$ then

$$
T(x)=\lim _{n \rightarrow \infty} T_{n}(x) \leqslant \lim _{n \rightarrow \infty} T_{n}(y)=T(y) .
$$

Corollary 3.9. Let $E$ and $F$ be vector spaces with an order unit, $\left\{T_{n}\right\}$ a sequence of weakly additive, order-preserving operators $T_{n}: E \rightarrow F$. If there exists a limit $\lim _{n \rightarrow \infty} T_{n}(x), x \in E$, then an operator $T: E \rightarrow F$ defined by (3.1) is also a weakly additive, order-preserving operator.

\section{Order-preserving variant of open mapping theorem}

Remind that a map $f: X \rightarrow Y$ of topological spaces is called open at $x_{0} \in X$ if for every open neighbourhood of $x_{0}$ in $X$ there exists an open neighbourhood $V$ of $f\left(x_{0}\right)$ in $Y$ such that $V \subset f(U)$. A map is open on a topological space $X$ if it is open at every point of $X$.

Lemma 4.1. Let $E$ and $F$ be vector spaces with an order unit, $T: E \rightarrow F$ a weakly additive, order-preserving onto operator. If $T$ is open at zero then it is open on all $E$.

Proof. Let for every neighbourhood $U=U\left(0_{E}, \varepsilon\right)$ of $0_{E}$ its image $T(U)=\{T(x): x \in U\}$ be open. We have $0_{F} \in T(U)$ as $T\left(0_{E}\right)=0_{F}$. Thence there exists an open neighbourhood $V=V\left(0_{F}, \delta\right)$ of $0_{F}$ such that $V \subset T(U)$.

Now let $x_{0} \in E$ be an arbitrary point and $U\left(x_{0}, \varepsilon\right)$ a neighbourhood of $x_{0}$ got by shifting $U\left(0_{E}, \varepsilon\right)$ on vector $x_{0}$. Besides let $V\left(T\left(x_{0}\right), \delta\right)$ be a neighbourhood of $T\left(x_{0}\right)$ got by shifting $V\left(0_{F}, \delta\right)$ on vector $T\left(x_{0}\right)$. The proof of the Lemma will finish if we show that the following diagram is true

$$
\begin{array}{rlr}
y \in V\left(T\left(x_{0}\right), \delta\right) & \stackrel{(1)}{\Longleftrightarrow} \quad y-T\left(x_{0}\right) \in V\left(0_{F}, \delta\right) \\
& & (2) \Downarrow \\
y \in T\left(U\left(x_{0}, \varepsilon\right)\right) & \stackrel{(3)}{\Longleftrightarrow} y-T\left(x_{0}\right) \in T\left(U\left(x_{0}, \varepsilon\right)\right) .
\end{array}
$$

The equivalence of the double inequalities $-\delta 1_{F}<y-T\left(x_{0}\right)<\delta 1_{F}$ and $T\left(x_{0}\right)-\delta 1_{F}<y<\delta 1_{F}+T\left(x_{0}\right)$ implies $(1)$. Since $V \subset T(U)$ we have (2). And the equivalence of the double inequalities $-\varepsilon T\left(1_{E}\right)<y-T\left(x_{0}\right)<\varepsilon T\left(1_{E}\right)$ and $T\left(x_{0}\right)-\varepsilon T\left(1_{E}\right)<y<\varepsilon T\left(1_{E}\right)+T\left(x_{0}\right)$ implies (3).

Thus for an arbitrary point $x \in E$ and its arbitrary neighbourhood $U=U(x, \varepsilon)$ there exists open neighbourhood $V=V(T(x), \delta)$ such that $V \subset T(U)$.

Remind that a metric $d$ on a vector space $E$ is invariant concerning to a shift of points of $E$ if $d(x+z, y+z)=d(x, y)$ for all $x, y, z \in E$. Define an order metric by the rule

$$
d(x, y)=\|y-x\|=\inf \left\{\lambda>0:-\lambda 1_{E}<y-x<\lambda 1_{E}\right\}
$$

It is easy to see that the following assertion holds.

Lemma 4.2. The order metric on a vector space with an order unit is invariant according to a shift of points.

Let $E$ and $F$ be vector spaces with an order unit. A product $E \times F$ over $\left(0_{E}, 0_{F}\right)$ becomes a vector space with an order unit if we will introduce to it coordinatewise operations of sum and multiplication by number

$$
\alpha\left(x_{1}, x_{2}\right)+\beta\left(y_{1}, y_{2}\right)=\left(\alpha x_{1}+\beta y_{1}, \alpha x_{2}+\beta y_{2}\right),
$$

and coordinatewise partially order

$$
\left(x_{1}, x_{2}\right) \leqslant\left(y_{1}, y_{2}\right) \Leftrightarrow\left(x_{1} \leqslant y_{1} \text { and } x_{2} \leqslant y_{2}\right) .
$$

Order norm on $E \times F$ is defined by the rule

$$
\left\|\left(x_{1}, x_{2}\right)\right\|=\inf \left\{\lambda>0:-\lambda\left(1_{E}, 1_{F}\right) \leqslant\left(x_{1}, x_{2}\right) \leqslant \lambda\left(1_{E}, 1_{F}\right)\right\} .
$$

Here $\left(1_{E}, 1_{F}\right)$ is one of inner points of $(E \times F)_{+}=E_{+} \times F_{+}$that is why without losing generality we assume $\left(1_{E}, 1_{F}\right)$ is an order unit in the product. Denote $1_{E \times F}=\left(1_{E}, 1_{F}\right)$.

Let $T: E \rightarrow F$ be a weakly additive, order-preserving operator. The set of all pairs $(x, T(x)), x \in E$, is called $a$ graph of $T$.

Lemma 4.3. Let $E$ and $F$ be vector spaces with an order unit, $1_{E}$ an order unit in $E, T: E \rightarrow F$ a weakly additive, order-preserving operator. Then the graph $G$ of $T$ is an A-subspace of $E \times T(E)$ with an order unit $1_{E \times T(E)}$.

Proof. We have $\left(0_{E}, 0_{F}\right) \in G \subset E \times T(E)$ since $T\left(0_{E}\right)=0_{F}$. Consider $\left(x_{1}, x_{2}\right) \in E \times T(E)$ and $\lambda \in \mathbb{R}$. Then

$$
\begin{aligned}
\left(x_{1}, x_{2}\right)+\lambda 1_{E \times T(E)} & =\left(x_{1}, T\left(x_{1}\right)\right)+\left(\lambda 1_{E}, \lambda 1_{T(E)}\right)=\left(x_{1}+\lambda 1_{E}, T\left(x_{1}\right)+\lambda 1_{T(E)}\right) \\
& =\left(x_{1}+\lambda 1_{E}, T\left(x_{1}\right)+\lambda T\left(1_{E}\right)\right)=\left(x_{1}+\lambda 1_{E}, T\left(x_{1}+\lambda 1_{E}\right)\right),
\end{aligned}
$$

i.e. $\left(x_{1}, x_{2}\right)+\lambda 1_{E \times T(E)} \in G$. 
Corollary 4.4. Let $E, F$ be vector spaces with an order unit, $1_{E}$ an order unit in $E, T: E \rightarrow F$ a weakly additive, order-preserving operator. Then the image $T(E)$ is A-subspace of $F$ if and only if $T\left(1_{E}\right) \in \operatorname{Int} F_{+}$.

Remark 4.5. Further, during the current section, without loss of generality, we will consider such weakly additive, order-preserving operators $T$ for which $T\left(1_{E}\right) \in \operatorname{Int} F_{+}$. Then we may assume that $T\left(1_{E}\right)$ is an order unit in $F$. Put $1_{F}=T\left(1_{E}\right)$.

At last, we will form a variant of the Open Mapping Theorem for weakly additive, order-preserving operators.

Theorem 4.6. Let $E$ be a complete space with an order unit, $F$ a vector space with an order unit, and $T: E \rightarrow F$ a weakly additive, order-preserving operator such that $T(E)=F$ and $F$ is a set of the second category. Then

(i) the map $T$ is open;

(ii) $F$ is a complete space with an order unit.

Proof. Let $U\left(0_{E}, \varepsilon\right)$ be an open neighbourhood. Then according to Remark 4.5 we have

$$
\begin{aligned}
& T\left(U\left(0_{E}, \varepsilon\right)\right)=\left\{T(x) \in F:-\varepsilon 1_{E}<x<\varepsilon 1_{E}\right\}= \\
& =\left\{T(x) \in F:-\varepsilon 1_{F}<T(x)<\varepsilon 1_{F}\right\}=\left\{y \in F: \text { there exists } x \in U\left(0_{E}, \varepsilon\right) \text { such that } y=T(x) \text { and }-\varepsilon 1_{F}<y<\varepsilon 1_{F}\right\}=U\left(0_{F}, \varepsilon\right) .
\end{aligned}
$$

It reminds to show that $(i i)$ takes place.

Let $\left\{y_{n}\right\} \subset F$ be a fundamental (Cauchy) sequence. Then for every $\varepsilon>0$ there exists a number $n$ such that at all $k \geqslant n, m \geqslant n$ the double inequalities

$$
-\varepsilon 1_{F}<y_{m}-y_{k}<\varepsilon 1_{F}
$$

hold. One may assume $\varepsilon=\frac{1}{n}$. Then $-\frac{1}{n} 1_{F}<y_{m}-y_{k}<\frac{1}{n} 1_{F}$. Since $T\left(U\left(0_{E}, \frac{1}{n}\right)\right)=U\left(0_{F}, \frac{1}{n}\right)$ there exists $x_{m}, x_{k} \in E$ such that $T\left(x_{m}\right)=y_{m}$, $T\left(x_{k}\right)=y_{k}$ and $-\frac{1}{n} 1_{E}<x_{m}-x_{k}<\frac{1}{n} 1_{E}$. So we have constructed a fundamental sequence $\left\{x_{n}\right\} \subset E$. By completeness of $E$ the sequence have a limit $x=\lim _{n \rightarrow \infty} x_{n}$. As $T$ is continuous from Proposition 3.3 we have $T(x)=\lim _{n \rightarrow \infty} T\left(x_{n}\right)=\lim _{n \rightarrow \infty} y_{n}$. Then $\lim _{n \rightarrow \infty} y_{n} \in T(E)=F$. Thus, $F$ is complete space with an order unit.

Remark 4.7. Note that open mapping principle for weakly additive, order-preserving operators it is impossible to form as the linear case. In the distinguishing from the linear case, weakly additivity and order-preserving of $T$, and being of $T(E)$ the second category set does not imply the equality $T(E)=F$. On the other hand $T(E)$ must not be open in $F$. At last, if $T$ is not onto in Lemma 4.1 then openness of $T$ at zero does not provide it openness on all the space.

Note that in linear topological spaces there is no open subspace different the whole space. But an $A$-subspace, distinguished from the subspace, may be open, closed or everywhere dense in the vector space with order unit.

Example 4.8. Let $L=\mathbb{R}^{2}=\left\{\left(x_{1}, x_{2}\right): x_{i} \in \mathbb{R}, i=1,2\right\}$ be the vector space with an order unit considered in Example 2.5. Then $L_{+}=\left\{\left(x_{1}, x_{2}\right) \in \mathbb{R}^{2}: x_{i} \geqslant 0, i=1,2\right\}$ is a positive cone in $L$. Fix $\mathbf{1}=(1,1) \in \operatorname{Int} \mathbb{R}_{+}^{2}=\left\{\left(x_{1}, x_{2}\right) \in \mathbb{R}^{2}: x_{1}>0, x_{2}>0\right\}$ as an order unit in it.

a) It is easy to see that the set $B=\left\{\left(x_{1}, x_{1}+a\right) \in \mathbb{R}^{2}:-1<a<1\right\}$ is an open (with respect to order topology) $A$-subspace, but $B \neq \mathbb{R}^{2}$.

b) Let $\mathbb{Q}$ be the set of rational numbers. Then $C=\left\{\left(x_{1}, x_{1}+r\right) \in \mathbb{R}^{2} ; r \in \mathbb{Q}\right\}$ is an everywhere dense $A$-subspace in $\mathbb{R}^{2}$.

c) The set $D=\left\{\left(x_{1}, x_{1}+a\right) \in \mathbb{R}^{2}:-1 \leqslant a \leqslant 1\right\}$ is a closed $A$-subspace in $\mathbb{R}^{2}$.

d) Define a map $T: \mathbb{R}^{2} \rightarrow \mathbb{R}^{2}$ by the rule

$$
T\left(x_{1}, x_{2}\right)=\left\{\begin{array}{lll}
\left(x_{1}, x_{1}-1\right), & \text { at } \quad x_{2} \leqslant x_{1}-1, \\
\left(x_{1}, x_{2}\right), & \text { at } \quad x_{1}-1<x_{2}<x_{1}+1, \\
\left(x_{1}, x_{1}+1\right), & \text { at } \quad x_{2} \geqslant x_{1}+1 .
\end{array}\right.
$$

It is easy to check that $T$ is a weakly additive map. Let us show that the map $T$ is order-preserving. It clear that $T$ is order-preserving on $B$ by $T=i d_{B}$

Let $x_{2} \geqslant x_{1}+1$. Take a vector $\left(y_{1}, y_{2}\right) \in \mathbb{R}^{2}$ such that $\left(x_{1}, x_{2}\right) \leqslant\left(y_{1}, y_{2}\right)$. The following three cases possible.

Case 1) $y_{2} \geqslant y_{1}+1$. Then

$$
T\left(x_{1}, x_{2}\right)=\left(x_{1}, x_{1}+1\right) \leqslant\left(y_{1}, y_{1}+1\right)=T\left(y_{1}, y_{2}\right) .
$$

Case 2) $y_{1}-1 \leqslant y_{2} \leqslant y_{1}+1$. Then $x_{1}+1 \leqslant y_{2}$. That is why

$$
T\left(x_{1}, x_{2}\right)=\left(x_{1}, x_{1}+1\right) \leqslant\left(y_{1}, y_{2}\right)=T\left(y_{1}, y_{2}\right) .
$$

Case 3) $y_{2} \leqslant y_{1}-1$. Then $x_{1}+1 \leqslant y_{1}-1$. Censequently

$$
T\left(x_{1}, x_{2}\right)=\left(x_{1}, x_{1}+1\right) \leqslant\left(y_{1}, y_{1}-1\right)=T\left(y_{1}, y_{2}\right) .
$$

Similarly, one may show that $T$ is order-preserving when $x_{2} \leqslant x_{1}-1$. Thus $T$ is order-preserving on the whole $\mathbb{R}^{2}$.

We have $T\left(\mathbb{R}^{2}\right)=D \neq \mathbb{R}^{2}$ through the operator $T$ is weakly additive and order-preserving, and the image $T\left(\mathbb{R}^{2}\right)$ is the second category. Clearly the image $T\left(\mathbb{R}^{2}\right)$ is closed in $\mathbb{R}^{2}$ and it is not open. Moreover $T$ is open at zero but it is not open on $\mathbb{R}^{2}$. Really for the open neighbourhood $U((2,4), 1)=\left\{\left(x_{1}, x_{2}\right) \in \mathbb{R}^{2}: 1<x_{1}<3,3<x_{2}<5\right\}$ of the point $(2,4) \in \mathbb{R}^{2}$ its image $T(U)=\left\{\left(x_{1}, x_{1}+1\right): 1<x_{1}<3\right\}$ is not open in $T\left(\mathbb{R}^{2}\right)$. 


\section{Order-preserving variant of Banach-Alaoglu theorem}

Let $E$ be a vector space with an order unit. Fix $1_{E}$ as an order unit. By $E_{+}^{W}$ we denote the set of all weakly additive, order-preserving functionals $f: E \rightarrow \mathbb{R}$. On $E_{+}^{W}$ define algebraic operations pointwise. Then $E_{+}^{W}-E_{+}^{W}$ turns to a vector space with an order unit. Denote $E^{W}=E_{+}^{W}-E_{+}^{W}$. Put $E^{O}=\left\{f \in E_{+}^{W}: f\left(1_{E}\right)=1\right\}$. Provide $E^{W}$ with the pointwise convergence topology. A collection of the sets of the view

$$
\left\langle f ; x_{1}, \ldots, x_{n} ; \varepsilon\right\rangle=\left\{g \in E^{W}:\left|f\left(x_{i}\right)-g\left(x_{i}\right)\right|<\varepsilon, i=1, \ldots, n\right\}
$$

forms a base of open neighbourhoods of $f \in E^{W}$, where $\varepsilon>0, x_{i} \in E, i=1, \ldots, n$.

The main result of the section is the following variant of the Banach-Alaoglu theorem for weakly additive, order-preserving functionals.

Theorem 5.1. If $V$ is a neighbourhood of zero in $E$ then the set

$$
K=\left\{f \in E^{O}:|f(x)| \leqslant 1 \text { for every } x \in V\right\}
$$

is a compact in the pointwise convergence topology.

Proof. Since neighbourhoods of zero are absorbing sets, for every point $x \in E$ there exists $\gamma(x) \in \mathbb{R}_{+}$such that $x \in \gamma(x) V$. That is why $|f(x)| \leqslant \gamma(x)$ for all $f \in E^{W}$ and $x \in E$. For every $x \in E$ denote $D_{x}=[-\gamma(x), \gamma(x)]$ and assume that $\tau$ is the Tychonoff topology in the product $P=\prod_{x \in E} D_{x}$. It is well known that $P$ is a Hausdorff compact space. By the construction we have $K \subset P \cap E^{W}$. We will show that $K$ is closed in $P$. Let $f_{0} \in P$ and $f_{0}=f_{0}^{+}-f_{0}^{-}$, where $f_{0}^{+}, f_{0}^{-} \in P \cap E_{+}^{W}$. Suppose $\left\{f_{\alpha}^{+}\right\} \subset P \cap E_{+}^{W}$ and $\left\{f_{\theta}^{-}\right\} \subset P \cap E_{+}^{W}$ are nets converging to $f_{0}^{+}$and $f_{0}^{-}$respectively. Then owing to Corollary 3.9 we have $f_{0}^{+}, f_{0}^{-} \in E^{W}$, and therefore $f_{0} \in E^{W}$. On the other hand $\left|f_{0}(x)\right|=\left|\left(f_{0}^{+}(x)-f_{0}^{-}(x)\right)\right| \leqslant \max \left\{\left|f_{0}^{+}(x)\right|,\left|f_{0}^{-}(x)\right|\right\} \leqslant \gamma(x)$ by $\left|f_{\alpha}^{+}(x)\right| \leqslant \gamma(x)$ and $\left|f_{\theta}^{-}(x)\right| \leqslant \gamma(x)$ for all $x \in E, \alpha$ and $\theta$. Therefore $\left|f_{0}^{+}(x)\right| \leqslant \gamma(x)$ for all $x \in E$ and $\left|f_{0}^{+}(x)\right| \leqslant 1$ so far as $x \in V$. It means that $f_{0} \in K$.

Corollary 5.2. $E^{O}$ is a compact in the pointwise convergence topology.

If $E$ is a separable vector space with an order unit then Theorem 5.1 improves as

Theorem 5.3. If $E$ is a separable vector space with an order unit, and $K$ is a compact (with respect to pointwise convergence topology) subspace of $E^{W}$ then $K$ is metrizable.

Proof. Let $\left\{x_{n}\right\}$ be countable everywhere dense subset of $E$. For every $f \in E^{W}$ put $M_{n}(f)=f\left(x_{n}\right)$. By the definition of pointwise convergence topology, every $M_{n}$ is a continuous function on $E^{W}$. If $M_{n}(f)=M_{n}\left(f^{\prime}\right)$ for all $n$ then continuous functions $f$ and $f^{\prime}$ coincide on everywhere dense subset. Thus $\left\{M_{n}\right\}$ is a countable family of continuous functions which separate points of the space $E^{W}$, in particular of $K$. Hence $K$ is metrizable as each Hausdorff compact space which has a countable sequence of real-valued functions separating its points is metrizable.

Corollary 5.4. If $E$ is separable vector space with an order unit then $E^{O}$ is a metrizable compact in the pointwise convergence topology.

\section{Acknowledgement}

The author would like to express gratitude to the reviewers, professors Mahmut Akyiğit and Fuat Usta for the revealed shortcomings, the specified remarks, corrections and useful advices.

\section{References}

[1] M. Grabisch, M. Roubens, Application of the Choquet Integral in Multicriteria Decision Making, In the book 'Fuzzy Measures and Integrals - Theory and Applications' (eds M. Grabisch, T. Murofushi, M. Sugeno), Physica Verlag, (2000), 348-374.

[2] T. Radul, Idempotent measures: Absolute retracts and soft maps, (2018), arXiv:1810.09140v1 [math.GN].

[3] W. Rudin, Functional Analysis (2nd edition), International Editions (McGraw-Hill Book Co - Singapore for manufacture and export), 1991.

[4] A. Peperko, Uniform boundedness principle for nonlinear operators on cones of functions, Hindawi J. Func. Spaces, 2018, Article ID 6783748, 5 pages, available at https://doi.org/10.1155/2018/6783748

[5] A. Zaitov, On extension of order-preserving functionals, Doklady Akademii Nauk Uzbekistan, 5 (2005), 3-7.

[6] A. Zaitov, Banach-Alaoglu theorem for order-preserving functionals, Theses of reports of the international scientific conference "Operator Algebras and Quantum Probability Theory", Tashkent, (2005), 81-83.

[7] A. Zaitov, Open mapping theorem for order-preserving operators, The collection of theses of the International conference of young scientists devoted 1000 to the anniversary of Mamun Academy of Khwarezm, Tashkent, (2006), 4-5.

[8] V. Paulsen, M. Tomforde, Vector spaces with an order unit, Indiana Univ. Math. J., 58(3) (2009), 1319-1359. 